\title{
Stability of Asynchronous Feedback-Interconnected Dissipative Systems
}

\author{
M. Lopez-Martinez, C. Canudas-de-Wit and F.R. Rubio
}

\begin{abstract}
This paper presents a method to analyze the stability of the feedback interconnection of a class of dissipative linear systems when the sampling associated to the feedback interconnection is asynchronous. We consider systems that are either Input/Output Strictly passive (IOSP), or systems which have bounded $L_{2}$-gains less than one. The analysis is performed by using the concept of MAximum Sampling time preserving Dissipation (MASD), for each interconnected system. We investigate the impact of using the scattering transformation in the computation of the MASD, and we provide a numerical algorithm (based on a set of LMI's) that allows to choose the most suitable configuration for the interconnection.
\end{abstract}

\section{INTRODUCTION}

This paper presents a method to analyze the stability of the feedback interconnection of a class of dissipative linear systems when the sampling associated to the feedback interconnection is asynchronous. The considered scenario is shown in Fig. 1. These types of interconnections are present in many application fields such as remotely-operated systems [1], interconnected vehicle control sub-systems, and more generally in component-based control design where synchronous exchange of information is not feasible.

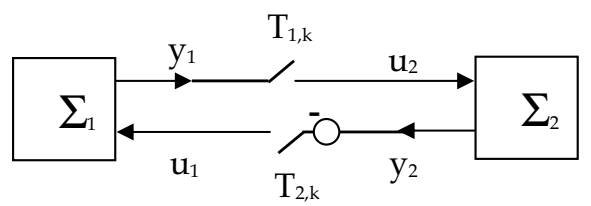

Fig. 1. Asynchronous Feedback Interconnection. System $\Sigma_{1}$ and system $\Sigma_{2}$ are feedback interconnected throughout the asynchronous, and possible time-varying, sampling $T_{1, k}$, and $T_{2, k}$.

It is well known that, the continuous time interconnection of two systems in feedback form is stable when each system posses certain passivity property, or satisfies the small gain condition (see [2], [3], [4]). As these two properties are fundamentally different (the first is phase shift sensible, while the second depends on its gain characteristics), it is expected that these conditions may behave differently when the output signals are sampled as shown in Fig. 1. In the framework of this work, the maximum sampling time preserving the stability of its original interconnection, may be different to each case.

Scattering transformation is a well known method whose change of variables is used to improve and/or to preserve the

M. Lopez-Martinez and F.R. Rubio are with the Department of systems and automation, University of Seville, Spain mlm, rubio@esi.us.es

C. Canudas-de-Wit is with the CNRS, at Gipsa-Lab, Grenoble, France carlos.canudas-de-wit@gipsa-lab.inpg.fr system original stability properties when the interconnecting signals are subject to delays. In addition, this transformation has been shown, in the continuous-time case [5], [6], [7], to imply a certain duality between passivity (IOSP) and $L_{2}$ gain (with $\gamma<1$ ) property. In these works, [5], [6], [7], the scattering transformation has been used in the context of continuous time system with fix or variable delays induced by the network, but the potential impact of an asynchronous sampling in the scenario shown in Fig. 2 has been scarcely studied. One of the objectives of this paper is to study how the scattering transformation impacts the maximum sampling time preserving the stability of its original interconnection.

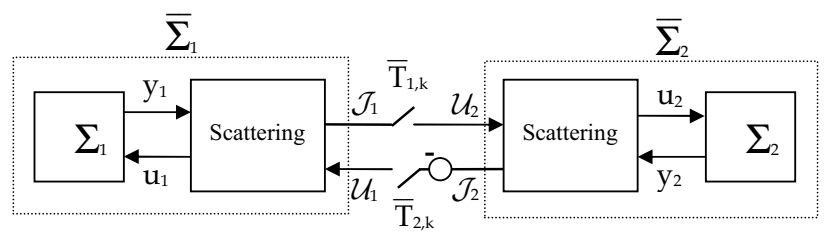

Fig. 2. Interconnection by means of scattering. Scattering may be used as a mean to improve the system stability under asynchronous sampled interconnection.

The lost of the original system properties such as dissipation and small gain, under the sampling process has been studied before. In [8], [9], [10], [11] it has been shown that positive realness can be lost in the process of discretization, and that in order to preserve the property of passivity in sampled systems, it is necessary that the outputs of these systems depends on their inputs, forcing the system relative degree to be zero. In [12] the authors have introduced a Lyapunov-based theory for asynchronous linear systems and shows how Lyapunov functions and controllers can be constructed by solving linear matrix inequalities (LMI). This theory was extended to nonlinear systems in [13]. In [14], the interconnection of passive asynchronous systems was studied, and control design method (based in a set of LMI's) was introduced. The LMI's were constructed using an Euler approximation of the derivative of the Lyapunov function, being the approach different from [15], [16], [17], where the approximation is introduced in the model.

This paper extends the results in [14], dealing only with passive system, to a more general dissipative systems, including $L_{2}$-gain stable systems. The objective is also to devise a general method to find an optimal way to interconnect a dissipative system that tolerates the maximum possible sampled time intervals. The method includes the possibility to use or not the scattering transformation, and allows us to devise a numerical algorithm able to compute the Maximum sampling time preserving stability of such an interconnection. 
The paper is organized as follows. In section II, we present the problem formulation for asynchronous feedbackinterconnected dissipative systems, and we also introduce some background on scattering theory with application to the interconnection of dissipative systems. The main results are given in Section III and IV. In Section III, we characterize the conditions required to preserve dissipation for asynchronous sampled systems, and in section IV, we provide necessary conditions to guarantee the stability of the interconnection. Our results are summarize in Section V.

\section{PROBLEM FORMULATION AND PRELIMINARIES}

Consider the feedback interconnection of two linear systems $\Sigma_{1}$ and $\Sigma_{2}$, as shown in Fig. 1, with each system $\Sigma_{i}$, of the following form:

$$
\begin{aligned}
\Sigma_{i}: & \dot{x}_{i}=A_{i} x_{i}+B_{i} u_{i} \\
y_{i} & =C_{i} x_{i}+D_{i} u_{i}
\end{aligned}
$$

subject to a general dissipative condition given by

$$
\dot{V}_{i}(x, u) \leq-\left(y_{i}^{\top} Q_{i} y_{i}+u_{i}^{\top} R_{i} u_{i}+2 y_{i}^{\top} S_{i} u_{i}\right)-\rho_{i} V_{i}(x)
$$

where $V_{i}(x)$ is a positive definite function, that can be chosen for linear systems as $V_{i}(x)=x_{i}^{\top} P_{i} x_{i}$.

We consider here two specific classes of systems characterized by the properties of matrices $Q_{i}, R_{i}$ and $S_{i}$ (see [18], [19]):

$\mathcal{C}_{1}$ : Input/Output Strictly Passive systems (IOSP); $Q=\delta I>$ $0, S=-\frac{1}{2} I$ and $R=\epsilon I>0$

$\mathcal{C}_{2}$ : Finite small $L_{2}$-gain systems $(\gamma<1) ; Q=I, S=0$ and $R=-\gamma^{2} I$

The problem considered in this paper is to find, for each system class $\mathcal{C}_{1}$, and $\mathcal{C}_{2}$ the maximum sampling times, such that their dissipative and finite small gain original properties are preserved, and thus to ensure that the interconnection is stable. To this aim, we also consider the the possibility to use the scattering transformation in the setup shown in Fig. 2, and compare this possibility to the one without the transformation. This combination leads to four cases; A, B, $\mathrm{C}$ and $\mathrm{D}$ shown in Table $\mathrm{I}$.

TABLE I

DIFFERENT CASES TO BE STUDIED IN THE PAPER.

\begin{tabular}{|l|c|c|}
\hline & $\begin{array}{c}\text { System class } \\
\mathcal{C}_{1}\end{array}$ & $\begin{array}{c}\text { System class } \\
\mathcal{C}_{2}\end{array}$ \\
\hline without scattering & $\mathrm{A}$ & $\mathrm{B}$ \\
\hline with scattering & $\mathrm{C}$ & $\mathrm{D}$ \\
\hline
\end{tabular}

There is a certain duality between these cases; a system that is IOSP (class $\mathcal{C}_{1}$ ) has its $L_{2}$-gain small than one after using the scattering transformation, $S$, and viceversa, a system with $L_{2}$-gain small than one (class $\mathcal{C}_{2}$ ) becomes IOSP after using the transformation $S$. This is discussed in detail next.

\section{A. Passivity and $L_{2}$-gain duality throughout scattering trans-} formation

A way to analyze the stability of the interconnection of two systems is via Passivity or $L_{2}$-gain. If both systems are passive or have their respective $L_{2}$-gain equal or less than one, then the interconnection is stable. However, the two properties are fundamentally different; a system can preserve its passivity property while is $L_{2}$-gain varies, while another can preserve its small gain property while the passivity is lost. This is illustrated by Fig.3.
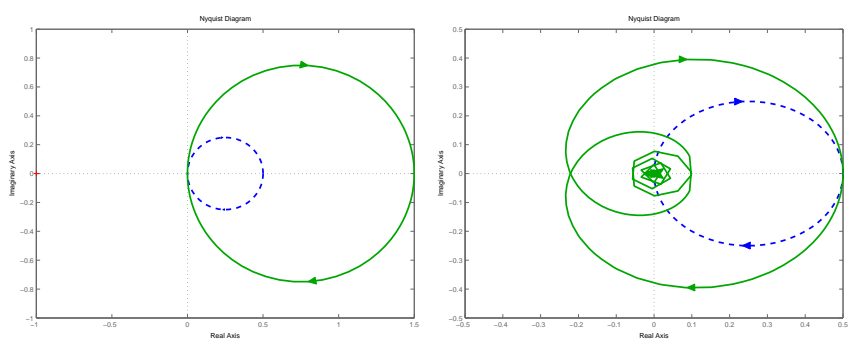

Fig. 3. Output passive system with different $L_{2}$-gains (left). System with different passivity properties preserving its $L_{2}$-gain (right).

Nevertheless, if the scattering transformation is used to interconnect the two sub-systems as in Fig. 2, i.e.

$$
u=\frac{1}{\sqrt{2}}(\mathcal{U}+\mathcal{J}), \quad y=\frac{1}{\sqrt{2}}(\mathcal{U}-\mathcal{J})
$$

where $u$ and $y$ are respectively the input and the output of each system, and $\mathcal{U}$ and $\mathcal{J}$ are the ones resulting from this transformation, it has been shown (see Proposition 2.3.4 in [2]) that: if and only if a system is IOSP from $u$ to $y$, then the resulting transformed system has a $L_{2}$-gain less than one from $\mathcal{U}$ to $\mathcal{J}$. This result can be easily understood following the next reasoning which will be useful for our next development.

Assume that we want the transformed system, $\bar{\Sigma}$, to have $L_{2}$-gain less than one. Therefore, it should hold that

$$
\dot{V}+\rho V<\frac{1}{2}\left(\mathcal{U}^{\top} \mathcal{U}-\mathcal{J}^{\top} \mathcal{J}\right)
$$

with $\rho \geq 0$. After substitution of (4) in (5), we get

$$
\dot{V}+\rho V-u y<0 \text {. }
$$

This inequality is equivalent to say that there exists,for the system $\Sigma$, a positive definite function, $w_{D}(u, y)$,

$$
w_{D}(u, y)=y^{\top} Q y+u^{\top} R u+2 y^{\top} S u>0
$$

where $Q>0$ and $R>0$, such that

$$
\dot{V}+\rho V-u y \leq-w_{D}(u, y) \text {. }
$$

To prove this, we substitute $y=C x+D u$ and define $\xi^{T}=\left[x^{T}, u^{T}\right]$ in the above inequality, where

$$
-w_{D}(u, y)=-\xi^{\top} M \xi \leq-\varepsilon \xi^{\top} \xi
$$

with

$$
M=\left(\begin{array}{cc}
C^{\top} Q C & C^{\top} Q D+C^{\top} S \\
D^{\top} Q C+S^{\top} C & R+D^{\top} Q D+D^{\top} S+S^{\top} D
\end{array}\right)>0
$$


and $\varepsilon=\lambda_{\min }(M)>0 \Leftrightarrow Q>0, \quad R>0$. Finally, It is important to remark that always it is posible to find $Q>0$ and $R>0$ such that $\varepsilon>0$ is small enough, which means that $\Sigma$ must be an Input and Output Strictly Passive (IOSP) system.

\section{B. Symmetry and modularity design in the scattering trans- formation}

As there are many possibilities to define the scattering transformation, we wish to select one that make the transformation of one side independent to the other (modular design). In addition, and from the original problem, as defined in Fig. 1, the original system inter-connection requires that $y_{1}=u_{2}$ and $u_{1}=-y_{2}$. Then, any possible transformation should then preserve this relation.

A good choice fulfilling such requirements is to define

$$
\mathcal{U}_{1}=\frac{1}{\sqrt{2}}\left(u_{1}+y_{1}\right), \quad \mathcal{J}_{1}=\frac{1}{\sqrt{2}}\left(u_{1}-y_{1}\right)
$$

for system $\Sigma_{1}$, and

$$
\mathcal{U}_{2}=-\frac{1}{\sqrt{2}}\left(u_{2}+y_{2}\right), \quad \mathcal{J}_{2}=-\frac{1}{\sqrt{2}}\left(u_{2}-y_{2}\right)
$$

for system $\Sigma_{2}$. It can be easily checked that this transformation give rise to the same transfer function,

$$
\bar{\Sigma}_{i}: \quad \bar{G}_{i}(s)=\frac{\mathcal{J}_{i}}{\mathcal{U}_{i}}=\frac{u_{i}-y_{i}}{u_{i}+y_{i}}=\frac{1-G_{i}(s)}{1+G_{i}(s)},
$$

in any of the two possible system coordinates.

Note that the transformed system $\bar{\Sigma}_{i}$ in terms of the new input $\mathcal{U}$ and the new output $\mathcal{J}$ can be written as

$$
\begin{aligned}
\bar{\Sigma}_{i}: & \dot{x}=\bar{A} x+\bar{B} \mathcal{U} \\
\mathcal{J} & =\bar{C} x+\bar{D} \mathcal{U}
\end{aligned}
$$

where $\bar{A} \doteq A+B \bar{C}, \quad \bar{B} \doteq B\left(\frac{1}{\sqrt{2}} I+\bar{D}\right)$, $\bar{C} \doteq-\sqrt{2}(I+D)^{-1} C$ and $\bar{D} \doteq(I+D)^{-1}(I-D)$.

The dissipative properties of the transform system can be expressed, using (3), as

$$
\dot{V} \leq-\left(\mathcal{J}^{\top} \bar{Q} \mathcal{J}+\mathcal{U}^{\top} \bar{R} \mathcal{U}+2 \mathcal{J}^{\top} \bar{S} \mathcal{U}\right)-\rho V,
$$

where the new dissipative matrixes are defined as

$$
\begin{aligned}
\bar{Q} & \doteq \frac{1}{2}(Q+R-2 S) \\
\bar{R} & \doteq \frac{1}{2}(Q+R+2 S) \\
\bar{S} & \doteq \frac{1}{2}\left(R^{\top}-Q^{\top}\right) .
\end{aligned}
$$

\section{ChARACTERIZATION OF THE MAXIMUM SAMPLING TIME PRESERVING DISSIPATION: MASD}

In this section we aim to characterize the maximum sampling time that preserve the system original dissipation properties. We will treat the general case of the dissipation property including the two class of systems $\mathcal{C}_{1}$, and $\mathcal{C}_{2}$, with and without the scattering transformation described in the previous section. The results will be presented as the solution of a LMIs guaranteing that the sampled system will satisfy the corresponding dissipative condition.

\section{A. Discrete-time system description}

We have to re-formulate our problem in the discrete time framework starting with the discrete time representation of the system $\Sigma_{i}$ in (1),

$$
\begin{aligned}
\Sigma_{i, k}: \quad x_{k+1} & =A_{k} x_{k}+B_{k} u_{k} \\
y_{k} & =C x_{k}+D u_{k},
\end{aligned}
$$

where $A_{k}=e^{A T_{k}}$ and $B_{k}=A^{-1}\left(A_{k}-I\right) B$. and the system $\bar{\Sigma}_{i}$ in (12),

$$
\begin{array}{r}
\bar{\Sigma}_{i, k}: \quad x_{k+1}=\bar{A}_{k} x_{k}+\bar{B}_{k} \mathcal{U}_{k} \\
\mathcal{J}_{k}=\bar{C} x_{k}+\bar{D} \mathcal{U}_{k} .
\end{array}
$$

Next, the equivalent definition of the dissipative property needs to be reformulated also in this discrete-time framework. One possible way is to make a discrete-time approximation of (3), or (13) according to the considered case, by using the approximation

$$
\dot{V} \approx \frac{V_{k+1}-V_{k}}{T_{k}},
$$

where $V_{k}=x_{k}^{\top} P x_{k}$, and proceeding as in previous section to write the associated discrete-time LMI, which can be used to find the maximum value for $T_{k}$, preserving its dissipative property.

Note that each of the two considered classes of system can be then evaluated by the proper substitution of the associated $(Q, R, S, \rho)$ values.

\section{B. Original system coordinates}

The dissipation inequality (3) evaluated in the setup shown in Fig. 1, gives the following LMI

$\left(\begin{array}{cc}A_{k}^{\top} \frac{P}{T_{k}} A_{k}-\frac{P}{T_{k}} & A_{k}^{\top} \frac{P}{T_{k}} B_{k} \\ B_{k}^{\top} \frac{P}{T_{k}} A_{k} & B_{k}^{\top} \frac{P}{T_{k}} B_{k}\end{array}\right)+$
$+\left(\begin{array}{cc}C^{\top} Q C+\rho P & C^{\top}(Q D+S) \\ \left(D^{\top} Q+S^{\top}\right) C & R+D^{\top} Q D+D^{\top} S+S^{\top} D\end{array}\right) \leq 0$.

which in a compact notation writes as $W\left(T_{k}\right)+Z \leq 0$, with $W\left(T_{k}\right)$ being the first matrix sampling dependent, and $Z$ the matrix capturing the particular dissipative property of the original system. Note that here the values of $(Q, R, S, \rho)$ , and $P$ are given from the original system properties, and then we only need to search for the maximum value of $T_{k}$ that verifies the LMI. Note also that from continuity of the solution, (there exist always a solution for infinitely small $T_{k}$ ), a limit solution for $T_{k}^{*}>0$ will always exist. The definition of MASD, $T_{k}^{*}>0$, follows then from this observation, i.e.

$$
T_{k}^{*}=\max _{T_{k}>0}\left\{T_{k}: W\left(T_{k}\right)+Z \leq 0\right\}
$$




\section{Using the Scattering transformation}

Our second option will be to perform a similar analysis in the transformed system coordinates after applying the previously presented scattering transformation, in the setup given by Fig. 2. Proceeding as before, but now using (13), we get

$$
\begin{aligned}
& \left(\begin{array}{cc}
\bar{A}_{k}^{\top} \frac{P}{T_{k}} \bar{A}_{k}-\frac{P}{T_{k}} & \bar{A}_{k}^{\top} \frac{P}{T_{k}} \bar{B}_{k} \\
\bar{B}_{k}^{\dagger} \frac{P}{T_{k}} \bar{A}_{k} & \bar{B}_{k}^{\top} \frac{P_{F}}{T_{k}} \bar{B}_{k}
\end{array}\right)+ \\
& +\left(\begin{array}{cc}
\bar{C}^{\top} \bar{Q} \bar{C}+\rho P & \bar{C}^{\top}(\bar{Q} \bar{D}+\bar{S}) \\
\left(\bar{D}^{\top} \bar{Q}+\bar{S}^{\top}\right) \bar{C} & \bar{R}+\bar{D}^{\top} \bar{Q} \bar{D}+\bar{D}^{\top} \bar{S}+\bar{S}^{\top} \bar{D}
\end{array}\right) \leq 0 .
\end{aligned}
$$

which in a compact notation writes as $\bar{W}\left(T_{k}\right)+\bar{Z} \leq 0$. The definition of MASD $\bar{T}_{k}^{*}>0$, follows as before, i.e.

$$
\bar{T}_{k}^{*}=\max _{T_{k}>0}\left\{T_{k}: \bar{W}\left(T_{k}\right)+\bar{Z} \leq 0\right\}
$$

Remark 1: Note that both formulations (18) and (20) yield similar forms of the LMIs, but with matrices that inside have different values. Therefore, the expected values for each of the MASD; $T_{k}^{*}$, and $\bar{T}_{k}^{*}$ will be different.

\section{Examples}

1) First-order IOSP system: This example compares the MASD obtained for a dissipative system $\Sigma$ of class $\mathcal{C}_{1}$ (Case A in Tab. I), with and without the scattering transformation. For this, consider the following input-output strictly passive (IOSP) system,

$$
G(s)=\frac{1}{s+1}+d
$$

and recall that the use of the scattering transformation, makes the transformed system $\bar{\Sigma}$ have $L_{2}$-gain less than one (Case $\mathrm{D}$ in table I). Figure 4 shows the evolution of the $T_{k}^{*}$ and the $\bar{T}_{k}^{*}$ for different values of the parameter $d$. It can be noticed that the resulting MASD is larger when the scattering transformation is used $\left(\bar{T}_{k}^{*}>T_{k}^{*}\right)$. The example reveals how the use of the scattering transformation improves over the one without transformation.

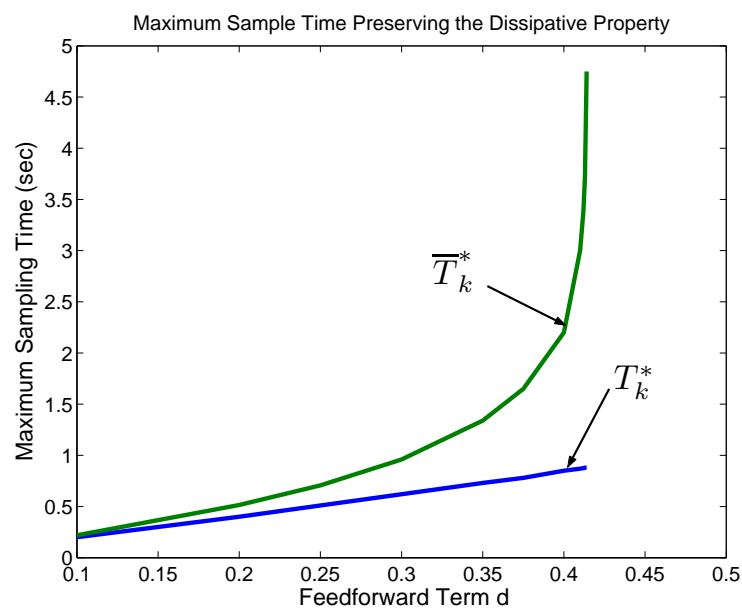

Fig. 4. Comparison of MASD for a first-order IOSP system with and without scattering transformation
2) Underdamped Second Order System: This example compares the MASD obtained for a dissipative system $\Sigma$ of class $\mathcal{C}_{2}$ (Case $\mathrm{B}$ in Tab. I), with and without the scattering transformation. Consider the system

$$
G(s)=\frac{K \omega_{n}^{2}}{s^{2}+2 \delta \omega_{n} s+\omega_{n}^{2}}
$$

with parameters $K=0.865, \omega_{n}=6$ and $\delta=0.5$. This system $\Sigma$ is not passive, but has $L_{2}$-gain equal to 0.999 .

Figure 5-(a) shows the $L_{2}$-gain versus the sampling time. It can be observed that the gain over-exceeds one at $T^{*}=0.46$.

The second option is to use the transform system using the scattering matrix. Because the duality indicated in previous section, the transform system $\bar{\Sigma}$ belongs to class $\mathcal{C}_{2}$ (Case $\mathrm{C}$ in Tab. I). Figure 5-(b) shows the $\lambda=\min (\mathcal{R} e\{G(j \omega)\})$ as a function of the sampling time. It can be observed that $\lambda>0$ for all sampling time, which shows that the best option (in terms of stability) to interconnect this system is by the scattering transformation.
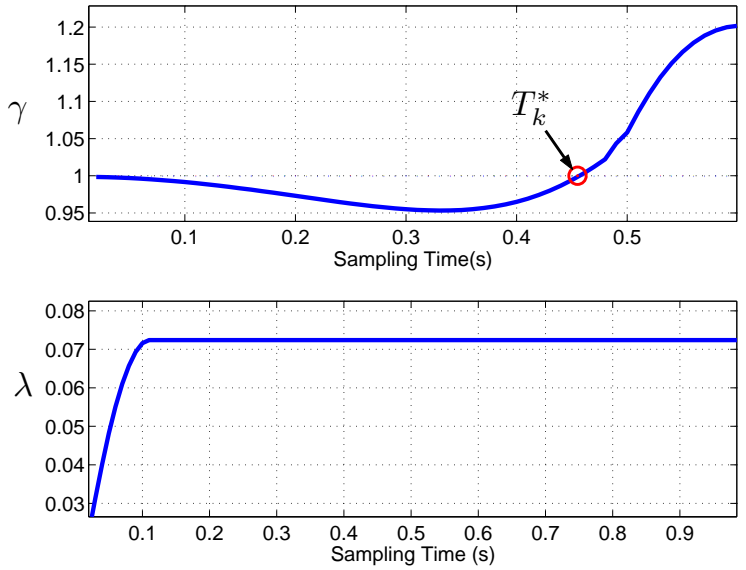

Fig. 5. Evolution of dissipative properties. (a)- Evolution of the $L_{2}$-gain $\gamma$ of $G(s)$ as a function of the sampling time, and (b)- Evolution of $\lambda=$ $\min (\mathcal{R} e\{G(j \omega)\})$ as a function of the sampling time.

One of the paper goal is to select the formulation that gives the larger MASD, while preserving the stability of the interconnection. Stability issues under such interconnection framework are studied next.

\section{STABILITY PROPERTIES OF ASYNCHRONOUS SAMPLED SYSTEMS}

Let us assume that each system ${ }^{1}$ is sampled using a zero order hold, and as before, that the sampling time-intervals $T_{2, k}$, and $T_{1, k}$ are not constant but are multiple-integers. For simplicity we consider the case in which $T_{2, k}=n_{k} T_{1, k}$, with $n_{k} \in\left\{1,2, \ldots, n_{\max }\right\}$, but similar results hold if $n_{k}$ is of the form $n_{k} \in\left\{1,1 / 2, \ldots, 1 / n_{\max }\right\}$.

\footnotetext{
${ }^{1}$ The notion of system here designs, either $\Sigma_{i}$, or $\bar{\Sigma}_{i}$, according with the considered case.
} 


\section{A. Stability conditions in the original system coordinates}

Proposition 1: Consider two dissipative systems ${ }^{2} \Sigma_{i}$, fulfilling each one its respective dissipative inequality (18), and interconnected asynchronously as shown in Fig. 1. Assume that there exists for each system, $\Sigma_{i}$, a MASD $T_{i}^{*}, i=1,2$, verifying

$$
T_{2, k}=n_{k} T_{1, k} \leq \min \left\{T_{1}^{*}, T_{2}^{*}\right\},
$$

Then, the following holds:

- the feedback asynchronous interconnection of two dissipative systems is asymptotically stable if the following LMI is satisfied

$$
\left(\begin{array}{cc}
Q_{1}+R_{2} & S_{1}-S_{2}^{\top} \\
S_{1}^{\top}-S_{2} & R_{1}+Q_{2}
\end{array}\right)>0
$$

- If $\rho_{i}>0, i=1,2$, then the feedback interconnection is also exponentially stable,

- For the two classes $\mathcal{C}_{1}$, and $\mathcal{C}_{2}$ of considered systems, the condition (23) holds, and hence the interconnection is asymptotically stable.

Proof: Assuming that there exist $T_{i}^{*}, i=1,2$, verifying $T_{2, k}=n_{k} T_{1, k} \leq \min \left\{T_{1}^{*}, T_{2}^{*}\right\}$, such that each sub-system $\Sigma_{i}$ fulfills its respective dissipative inequality, implies that that both systems must verify a dissipative property for the smaller sampling time, $T_{1, k}$, that is

$\Sigma_{1}: V_{1, k+1}-V_{1, k} \leq$

$\leq-T_{1, k}\left(y_{1, k}^{\top} Q_{1} y_{1, k}+u_{1, k}^{\top} R_{1} u_{1, k}+2 y_{1, k}^{\top} S_{1} u_{1, k}+\rho_{1} V_{1, k}\right)$

$\Sigma_{2}: V_{2, k+1}-V_{2, k} \leq$

$\leq-T_{1, k}\left(y_{2, k}^{\top} Q_{2} y_{2, k}+u_{2, k}^{\top} R_{2} u_{2, k}+2 y_{2, k}^{\top} S_{2} u_{2, k}+\rho_{2} V_{2, k}\right)$

As both systems are feedback interconnected it follows that $u_{2, k}=y_{1, k}$ and $y_{2, k}=-u_{1, k}$. Taking as a Lyapunov function candidate, $V_{k}=V_{1, k}+V_{2, k}$, the rate of change of this function $\Delta V_{k}=V_{k+1}-V_{k}$ is given as

$\Delta V_{k}=V_{1, k+1}-V_{1, k}+V_{2, k+1}-V_{2, k} \leq$

$-T_{1, k}\left(y_{1, k}^{\top} Q_{1} y_{1, k}+u_{1, k}^{\top} R_{1} u_{1, k}+2 y_{1, k}^{\top} S_{1} u_{1, k}+\rho_{1} V_{1, k}+\right.$

$\left.+u_{1, k}^{\top} Q_{2} u_{1, k}+y_{1, k}^{\top} R_{2} y_{1, k}-2 u_{1, k}^{\top} S_{2} y_{1, k}+\rho_{2} V_{2, k}\right)<0$

introducing $\bar{\rho} \doteq \min \left(\rho_{1}, \rho_{2}\right)$ yields,

$$
\begin{aligned}
\Delta V_{k} & \leq-T_{1, k}\left[y_{1, k}^{\top}\left(Q_{1}+R_{2}\right) y_{1, k}\right. \\
& +u_{1, k}^{\top}\left(R_{1}+Q_{2}\right) u_{1, k} \\
& \left.+2 y_{1, k}^{\top}\left(S_{1}-S_{2}^{\top}\right) u_{1, k}+\bar{\rho} V_{k}\right]<0
\end{aligned}
$$

It follows straightforward that if $\bar{\rho}=0$ asymptotic stability can be reached if the two systems are ZSD, and the condition (23) hold, insuring that $\Delta V_{k} \leq 0$.

The second item in the proposition is simple to check by observing that if $\bar{\rho}>0$, then $\Delta V_{k}$ is an strictly decreasing function, and hence the interconnection become exponentially stable.

Finally, the last item is easy to check by simple substitution of the definition of the $\left(Q_{i}, R_{i}, S_{i}, \rho_{i}\right)$ in the equation (23).

\footnotetext{
${ }^{2}$ For completeness we also assume that both systems are zero state detectable (ZSD).
}

The proposition can be particularized for every kind of dissipative systems, i.e. in terms of passivity or in terms of $L_{2}$-gain. The proposition is also valid for systems interconnected with the scattering transformation, as shown next.

\section{B. Stability conditions using scattering transformation}

Proposition 2: Consider two dissipative systems ${ }^{3} \bar{\Sigma}_{i}$, fulfilling each one its respective dissipative inequality (20), and interconnected asynchronously by means of a scattering transformation as shown in Fig. 2. Assume that there exists for each system, $\bar{\Sigma}_{i}$, a MASD $\bar{T}_{i}^{*}, i=1,2$, verifying

$$
\bar{T}_{2, k}=n_{k} \bar{T}_{1, k} \leq \min \left\{\bar{T}_{1}^{*}, \bar{T}_{2}^{*}\right\},
$$

Then, the following holds:

- the feedback asynchronous interconnection of two dissipative systems is asymptotically stable if the following LMI is satisfied

$$
\left(\begin{array}{cc}
\bar{Q}_{1}+\bar{R}_{2} & \bar{S}_{1}-\bar{S}_{2}^{\top} \\
\bar{S}_{1}^{\top}-\bar{S}_{2} & \bar{R}_{1}+\bar{Q}_{2}
\end{array}\right)>0,
$$

- If $\rho_{i}>0, i=1,2$, then the feedback interconnection is also exponentially stable,

- For the two classes $\mathcal{C}_{1}$, and $\mathcal{C}_{2}$ of considered systems, the condition (25) holds, and hence the interconnection is asymptotically stable.

Proof: The proof is similar to the one in Proposition 1, and then it is omitted.

Remark 2: Substituting (14) in (25) the LMI can be expressed in terms of the original dissipative properties of the systems with no scattering transformation.

Remark 3: The condition given by the two previous propositions, can be also used in an inverse manner to find for the most appropriate maximum sampling time ensuring stability. Besides, it may be possible also to use this analysis for control design along the same lines than in the works of [14].

\section{Example}

Consider the interconnections of two systems of the form (22) as described in the Example III-D.2. The goal of this example is to evaluate by simulation the interconnection behavior for the two possible scenarios shown in Fig. 1 and Fig. 2, with the addition of a reference step signal applied to the system $\Sigma_{1}$ to better visualize the system transient responses.

The first simulation tests are done by the proper selection of the sampling time validating the stability conditions: $T_{k}=0.3 \mathrm{~s}$, which implies that $T_{k}<T_{k}^{*}<\bar{T}_{k}^{*}$. Figure 6-(a) shows the system outputs corresponding to the interconnection in Figure. 1 (Case B in Tab. I), and Figure 6-(b) the system output using the scenario shown in Figure 2 (Case $\mathrm{C}$ in Tab. I), including the scattering transformation. It can be seen how the inclusion of the scattering transformation increases the achieved performance.

\footnotetext{
${ }^{3}$ Both systems are assumed to be zero state detectable (ZSD).
} 
(a)
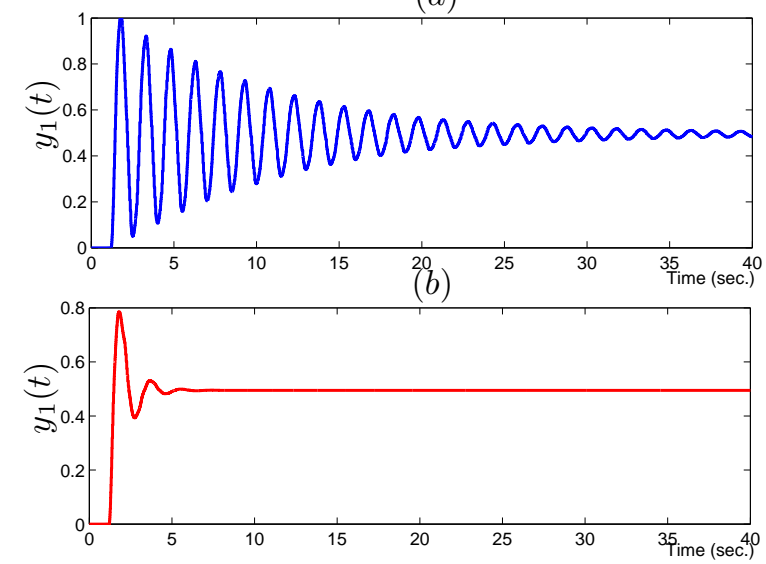

Fig. 6. Time-evolution of the system output $y_{1}(t)$. Sampling time fulfilling the stability conditions for both cases: $(a)$ without the use of the scattering transformation, and $(b)$ using the scattering transformation.

$(a)$

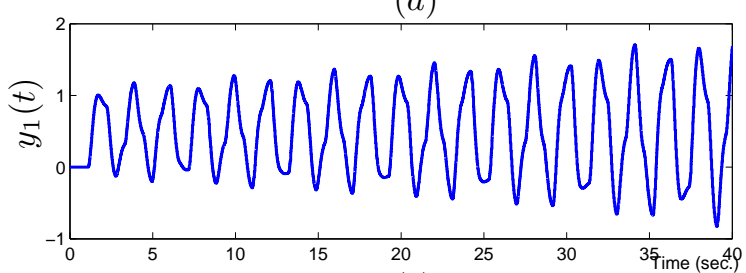

(b)

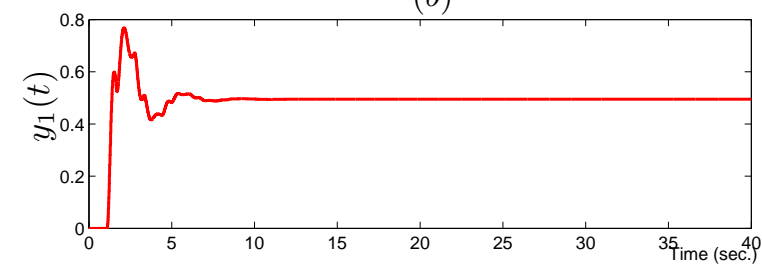

Fig. 7. Time-evolution of the system output $y_{1}(t)$ with $T_{k}^{*}<T_{k}<\bar{T}_{k}^{*}$. The case of sampling time not fulfilling the stability conditions is shown in (a) which does not the use of the scattering transformation. The case $(b)$ using the scattering transformation is stable and has a good performance.

The second example, shown in Figure 7, repeat the previous simulation but now with $T_{k}=0.55 \mathrm{~s}$. This sample time selection implies that $\left(T_{k}^{*}<T_{k}<\bar{T}_{k}^{*}\right)$. As the computation in the Example III-D.2 have shown, the use of the scattering matrices allows for larger sampling interval for stability, but also an improvement in the system transient behavior.

\section{CONCLUSIONS}

In this paper we have presented a method to analyze the stability of the feedback interconnection of a class of linear systems when the sampling is asynchronous. The analysis has been made taking into account dissipatedness properties of the original continuous-time system.

We have characterized specific conditions in terms of LMIs allowing to compute the larger sampling time interval preserving the original system dissipative properties. For that, we have also considered the possibility of using the scattering transformation. This transformation allows, in some cases, to improve the system stability over the situation where it is not used, because longer sampling time intervals can thus be tolerated.

We have presented stability conditions for the asynchronous interconnection of two general dissipative systems. The conclusions are extended to the case of using the scattering transformation in the interconnection. Finally, we have introduced a numeric procedure for choosing an optimal way to interconnect a dissipative system.

\section{ACKNOWLEDGMENTS}

The authors gratefully acknowledge MEC (Ministerio de Educación y Ciencia español) for founding this work under grants PR2007-0282 and DPI2007-64697, and CNRS (Centre National de la Recherche Scientifique, France) and INRIA-RA for allowing that the stay of the first author was possible.

\section{REFERENCES}

[1] P. Hokayem and M. Spong, "Bilateral teleoperation: An historical survey", Automatica, December 2006.

[2] A. J. van der Schaft, " $L_{2}-$ Gain and Passivity Techniques in Nonlinear Control”. Ed. Springer-Verlag. Berlin (1999).

[3] H. Khalil, Nonlinear control systems, Third Edition, 2001. London, UK.

[4] B. Brogliato, R.Lozano, B. Maschke and O. Egeland, "Dissipative Systems Analysis and Control". Theory and Applications, 2nd ed. London: Springer-Verlag, 2007.

[5] T. Matiakis, S. Hirche and M. Buss, "The Scattering Transformation for Networked Control Systems". Proceedings of the Conference on Control Applications, 2005.

[6] T. Matiakis, S. Hirche and M. Buss, "Independent-of-Delay Stability of Nonlinear Networked Control Systems by Scattering Transformation". Proceedings of the American Control Conference, 2006.

[7] N. Chopra and M. Spong,"Delay-Independent Stability for Interconnected Nonlinear Systems with Finite $L_{2}$ Gain", Proceedings of the Conference on Decision and Control, 2007.

[8] W.Lin and C.I. Byrnes. "Passivity and absolute stabilization of a class of discrete-time nonlinear systems". Automatica, Vol.31, N.2, pp.263267. 1995.

[9] M.De la Sen."Relationships between positive realness of continuos transfer functions and their digital counterparts". Electronic letters, Vol.35, N.16, pp. 1298-1299. 1999.

[10] M.De la Sen."Preserving positive realness through discretization". Proceedings of the American Control Conference, Chicago. 2000.

[11] S. Zhou, J. Lam, and G. Feng, "New characterization of positive realness and control of a class of uncertain polytopic discrete-time systems", Systems \& Control Letters, vol. 54, pp. 417427, November 2005.

[12] A. Hassibi, S. Boyd, and J. How, "Control of asynchronous dynamical systems with rate constraints on events", CDC'99, Phoenix, USA.

[13] D. Muñoz de la Peña and P.D. Christofides. "Stability of nonlinear asynchronous systems". CDC'07. New Orleans, L.A., USA.

[14] J.R.Cueli and C.Canudas-de-Wit. "Passivity of Interconnected Asynchronous Discrete-Time Systems". Submitted to World Ifac Congress, 2008

[15] D. Laila, D. Nesic, and A. Teel, "Open and closed loop dissipation inequalities under sampling and controller emulation”, European Journal of Control, 2002.

[16] D. Nesic, and A. Teel, "Stabilization of sampled-data nonlinear systems via backstepping on their Euler approximate model", Automatica, vol. 42, pp. 1801-1808, 2006.

[17] R. Rohrer and H. Nosrati, "Passivity considerations in stability studies of numerical integration algorithms", IEEE Transactions on Circuits and Systems, vol. CAS-28, no. 9, pp. 857866, September 1981.

[18] V.Chellaboina and W.Haddad. "Exponentially Dissipative Nonlinear Dynamical Systems: A Nonlinear Extension of Strict Positive Realness". Proceedings of the American Control Conference, Chicago. 2000 .

[19] E. Navarro-Lopez, "Several dissipativity and passivity implications in the linear discrete time-setting", Mathematical Problems in Engineering, vol. 6, pp. 599616, 2005. 